Esta obra está bajo una Licencia Creative Commons

Atribución-NoComercial-Compartirlgual 4.0 Internacional

Transversalización de la problemática de género y violencias en el Curso Introductorio, FCNyM, UNLP.

Relato de una experiencia de abordaje

Andrea Dipolitto, Florencia Lago

Trayectorias Universitarias, 7 (13), e070, 2021

ISSN 2469-0090 | https://doi.org/10.24215/24690090e070

https://revistas.unlp.edu.ar/TrayectoriasUniversitarias

Universidad Nacional de La Plata

La Plata | Buenos Aires | Argentina

\title{
Transversalización de la problemática de género y violencias en el Curso Introductorio, FCNyM, UNLP. Relato de una experiencia de abordaje
}

\section{Report of an experience of approaching the mainstreaming of gender and violence problem in the Introductory Course, FCNyM, UNLP}

\section{Andrea Dippolito}

https://orcid.org/0000-0001-7817-8368

adippolito@gsuite.fcnym.unlp.edu.ar

Facultad de Ciencias Naturales y Museo |

Universidad Nacional de La Plata | Argentina

\section{Florencia Lago}

https://orcid.org/0000-0002-5310-4634

florlago@gsuite.fcnym.unlp.edu.ar

Facultad de Ciencias Naturales y Museo |

Universidad Nacional de La Plata | Argentina

\section{RESUMEN}

En el presente trabajo, pretendemos relatar la experiencia de intervención de las y los ingresantes a la Facultad de Ciencias Naturales y Museo (FCNyM), transversalizando la perspectiva a lo largo del curso e identificándose en una acción puntual con motivo de la conmemoración internacional del 8M.

Como estrategia, se trabajó desde el inicio de la inscripción en la Facultad con diferentes materiales y estrategias para poner en evidencia la necesidad de considerar estas problemáticas como vinculadas a la accesibilidad e inclusión en los estudios superiores.

Optimizamos para pensar su diseño, información, contenidos y reflexiones compartidas en el marco del STPG, de la Dirección de Políticas de Género, DDHH UNLP realizado entre noviembre y diciembre de 2021.

Es así que disponiendo del aula virtual y sus posibilidades de llegada a la totalidad de la matrícula, en un entorno seguro, respetuoso y cuidado, plasmamos en una actividad conmemorativa una diversidad de cuestiones trabajadas y abordadas en cursos anteriores.

Esperamos que este artículo nos permita profundizar y compartir nuestras reflexiones, inquietudes y desafíos sobre las prácticas de transversalización de las políticas de inclusión y estrategias de acompañamiento en la Educación Superior.

PALABRAS CLAVE Educación Superior, género, violencias, estrategias. 
KEY WORDS

Higher Education, gender,

violence,

strategies.

\section{SUMMARY}

In this paper, we intend to relate the experience of the FCNyM entrants, mainstreaming the perspective throughout the course and identifying it in a specific action on the occasion of the international commemoration of the $8 \mathrm{M}$.

As a strategy, we worked from the beginning of enrollment in the Faculty with different materials and strategies to highlight the need to consider these problems as linked to accessibility and inclusion in higher education.

We optimize to think about your design information, content and reflections shared within the framework of the STPG, of the Dirección de Políticas de Género DDHH, UNLP

Thus, having the virtual classroom and its possibilities of reaching the entire enroIlment, in a safe, respectful and caring environment, we capture in a commemorative activity a diversity of issues worked on and addressed in previous courses.

We hope that this article allows us to deepen and share our reflections, concerns and challenges about the practices of mainstreaming inclusion policies and support strategies in Higher Education 
El espacio de Ingreso de la FCNYM (incluido en el Trayecto de Ingreso y seguimiento) considera como espacio inicial un Curso Introductorio de cumplimiento obligatorio (presencial adaptado a la virtualidad desde 2020) con objetivos de inserción, integración y orientación (Dippolito et al, 2017).

La mirada de inserción e integración ha sido de inclusión, sosteniendo estrategias diagnósticas en todas las actividades y espacios de participación y es así que parafraseando a Untoiglich (2013), consideramos que los diagnósticos en el Ingreso a la universidad se deben escribir con lápiz, considerando las múltiples y diversas situaciones devenidas de los recorridos individuales así como de las formas heterogéneas y variables con las cuales transitan sus primeros años en los estudios universitarios.

Trabajar con ingresantes nos convocó a reformular estrategias vinculadas a la problemática de género de manera continua, tal vez verbalizando y sin poder nombrar.

Las estrategias políticas y pedagógicas en el marco de las actividades del Curso Introductorio, se construyeron en el consenso, a partir de acciones que se consolidan a partir de prácticas instituidas relacionadas; las que a su vez retroalimentaban acciones. Su desarrollo se dio en diferentes esferas dentro del curso, que interactúan y se ensamblan entre sí, conformando tres dimensiones: Equipo coordinador (Coordinación General y Coordinadores de Módulo), Coordinación General y Dirección de Enseñanza, y Equipos docentes de Módulo.

Debates, conversaciones, lecturas y sobre todo escucha a quienes se acercaban a comentar alguna situación. Respeto y cautela ante las observaciones de aula, tanto las explícitas como aquellas en las cuales era posible definir, si atendían a supuestos docentes o situaciones que demandaban atención o intervención. Estas cuestiones definieron acciones puntuales e inmediatas, necesarias para acompañar a ingresantes y para diseñar estrategias de intervención que parecían 
novedosas o de ocurrencia sorpresiva, aunque consideramos que lo sorpresivo era nuestra capacidad cada vez mayor de observar y acompañar aquellas demandas que surgían de les ingresantes, y que interpelaban a los equipos docentes y a la Institución.

Las situaciones se hicieron más visibles, quizás porque podíamos identificarlas e interpretarlas, quizás porque se habilitaba la circulación de la palabra desde el inicio empezando por el lenguaje utilizado en la inscripción, el recibimiento y presentación del Curso. Las consignas se debatieron dentro de los equipos docentes teniendo en cuenta la inclusión y la perspectiva de género. La escritura de los cuadernillos, de los correos electrónicos y de la información en AulasWeb (soporte virtual en 2020 y 2021), utilizadas como aula extendida desde 2015, (Lago y Dippolito, 2016) se realizó utilizando progresivamente un lenguaje que no vulnere derechos, en su gramática y sintaxis.

\section{Las consignas se debatieron dentro de los equipos docentes teniendo en cuenta la inclusión y la perspectiva de género. La escritura de los cuadernillos, de los correos electrónicos y de la información en AulasWeb (soporte virtual en 2020 y 2021), utilizadas como aula extendida desde 2015, (Lago y Dippolito, 2016) se realizó utilizando progresivamente un lenguaje que no vulnere derechos, en su gramática y sintaxis.}

Atendiendo a que el lenguaje representa una herramienta inclusiva respecto de las violencias, no sólo la de género. Coincidimos con Cynthia Ottaviano (2020) quien afirma que "(...) A través del lenguaje, se construyen imágenes de las personas y grupos sociales, y se transmiten y refuerzan los estereotipos y roles de género, reproduciendo las relaciones asimétricas e inequitativas que se dan en la sociedad. El lenguaje, tal como lo vivimos en la actualidad, contribuye a la reproducción del paradigma patriarcal heteronormativo excluyente y, por ende, cada persona puede reproducir expresiones discriminatorias, estereotipadas o estigmatizantes."

Las situaciones habían existido siempre, solo que aumentaba su expresión, su verbalización o tal vez, nuestra mirada permitía reconocerlas. Quienes ingresan nos interpelan desde su experiencia, desde su militancia o desde su necesidad entre otras dimensiones. su expresión, su verbalización o tal vez, nuestra mirada permitía reconocerlas. Quienes ingresan nos interpelan desde su experiencia, desde su militancia o desde su necesidad entre otras dimensiones. 
Fue necesario también el sostén hacia el interior del equipo de coordinadoras en diferentes situaciones que nos atraviesan y nos interpelan para poder tomar decisiones y fortalecernos como equipo al generar esas estrategias.

Es así que fuimos contando al mismo tiempo con políticas nacionales y provinciales a las cuales la UNLP y consecuentemente la FCNyM adhirieron, generando espacios de formación, asesoramiento y construcción de espacios de acompañamiento.

Nos convoca poner en contexto la perspectiva de género y violencias Intentando hacer un relato histórico de su incorporación formal en el Curso Introductorio de la FCNyM, UNLP. Es difícil encontrar el punto de inicio, pensando en que siempre fue una mirada presente y no podemos desvincularlo de lo individual, subjetivo. Quizás es más sencillo comenzar por el momento en que estas políticas comenzaron a formar parte de la currícula.

- Taller de género para docentes del Cl 2018, 2019 (Coordinado por algunes Coordinadores del Curso y la Coordinadora General)

- Conversatorio y producción de documentos 2019, 2020 y 2021 (Coordinadores y UDAV FCNYM)

- Documentos de género construidos en consenso 2020 y 2021

- Capacitación coordinaciones y equipos Docentes Diplomados en Prosecretaria DDHH UNLP diciembre de 2019

- Mirando desde el género las carreras de la Universidad. Video Nexos 2018 (asesoría de contenidos y participación del Jefe de Ingreso J. M. Luis)

- Creación de la UDAV /FCNyM agosto 2018

- Taller para ingresantes 2019 y 2020

- Intervención de género con ingresantes 2021

Transversalización de la perspectiva crítica de género en las políticas de ingreso universitario, Seminario de posgrado, Dirección de políticas de género, DDHH, UNLP.

La propuesta para el Curso Introductorio 2021 tuvo el objetivo de reformular y adecuar el documento construido en ediciones anteriores, a su aplicación en entornos educativos digitales (Dippolito, 2020). Contábamos como insumo extra con el recursero de la Dirección de Género y Diversidad UNLP, accesible en los espacios EAD-UNLP, disponible tanto para docentes como para ingresantes. El documento se vio enriquecido en su contenido e incluyó nuevos debates en su construcción. La forma de trabajarlo fue desde los equipos docentes de Módulo, con formato de construcción colaborativa, en comisiones y luego por Módulo de Antropología, Biología y Geología. El documento de Módulo fue revisado y consensuado en el equipo Coordinador. A diferencia de otras ediciones se incluyó en el espacio de debate, la necesidad de proponer actividades y visiones sobre la manera de intervenir en el 8M. Es de mencionar que la inscripción fue record para la FCNyM numéricamente y que la matrícula inscrita por primera vez a 
una carrera de la FCNyM se distribuyó de la siguiente manera: Masculinxs: 378, Femeninxs: 460 tomando en cuenta la información obtenida en el sistema de Inscripción.

Como ejemplo mencionamos el Documento generado por el equipo docente del módulo de Biología que abordó los siguientes ítems: Marco Normativo. Naturalizar la perspectiva. Trabajo con estudiantes. Trabajo entre equipos docentes. Abordaje de situaciones. Propuesta de intervención 8M. Consideraciones finales (Equipo docente Módulo Específico Área de Biología, CIFCNyM 2021)

\section{PROPUESTA INTERVENCIÓN 8M:}

En la dinámica académica de la UNLP, la fecha se incluye en los espacios curriculares iniciales o de ingreso. Es sabido que en presencialidad se resuelve a través de facilidades académicas $u$ otras medidas que definen las Facultades autónomamente. En el Ingreso se ponen en tensión (evidenciado nuevamente en esta propuesta) las cuestiones propias de la conmemoración y las acciones colectivas que se difunden (paro internacional de mujeres, normas institucionales, derecho al paro, ¿hacen paro solo las mujeres? entre otras)

Esta cuestión lejos de quedar como discusión saldada nos lleva a un conflicto/tensión en el cual los y las docentes de ingreso nos vemos inmersas y que hemos diagnosticado como problemática específica propia.

Les ingresantes normalmente arriban al 8 de marzo, iniciando el afianzamiento al nuevo espacio de la vida universitaria, establecen sus primeros vínculos con estudiantes y docentes, así como con los diferentes actores institucionales. Sabemos de la diversidad de situaciones desde los desarraigos hasta la sensación de que han ingresado a un espacio donde inician un tránsito autónomo, donde quizás puedan autopercibirse como desean, manifestarse según sus necesidades y demandas, elegirse y elegir (si hay una palabra que define cabalmente a los ingresos es elección y la acompañaríamos con decisión).

\section{Los momentos de conmemoración internacional, en este caso del $\mathbf{8 M}$, tensionan y sensibilizan y es así que hace ya varios años la máxima tensión se expresa en la dualidad de estar en el aula e intervenir para poder acompañar o participar de las actividades. Actividades a las cuales les ingresantes se sienten convocades de manera heterogénea.}

Estas tensiones están lejos de haber sido resueltas y es entonces que consensuamos en optimizar la instancia de virtualidad diseñando una actividad Ad Hoc.

El 8M: "El aula de Curso Introductorio se silenció", todas los espacios, actividades, foros, materiales de lectura etc. se ocultaron y se generó un recurso nuevo. Esta intervención fue de carácter institucional, acor- 
dada con todas y todos los actores participantes en el espacio virtual del Curso y la gestión de la Facultad.

Se puso a disposición un solo espacio (rompiendo todo el esquema de funcionamientos por Módulos y Comisiones) al cual todes y todos podían ingresar. Allí se adjuntaron y se hicieron visibles los siguientes materiales;

Enlaces a videos y textos que historizaban y ponen en contexto la conmemoración. Video de presentación de la Lic. Bárbara Di Santo y presentación UDAV, FCNyM:

https://drive.google.com/file/d/1ad_IRsdFkMWJpJMdMETkFKj6yGylve1-/view?usp=sharing.

Video de Canal Encuentro vinculado al grooming http://encuentro. gob.ar/programas/133843

y un Foro de tareas con dos consignas generales:

Foro 1: Estamos trabajando en total virtualidad: ¿Qué formas de violencia podés reconocer en los diferentes entornos que utilizas para participar de las actividades del Curso?

Foro 2: Si te parece que una condición que está ocurriendo en una clase (entre estudiantes, entre docentes o entre docentes y estudiantes) puede contener un acto de violencia, puede pasarte a vos o a un compañere. ¿Cuál te parece que es la mejor manera de actuar?

No hubo actividad docente durante la jornada, pero la Coordinación General y las Coordinadoras de Biología y Antropología, nos mantuvimos en el espacio y es así que intervinieron en los foros 47 estudiantes (de los 621 matriculades actives en el aula). Como respuestas pudimos ver que agradecieron el espacio, se expresaron con libertad, manifestaron sus dudas, que fueron posteriormente incluidas en los espacios de trabajo. Surgieron también situaciones de abordaje que fueron derivadas a la UDAV. La reformulación y análisis de las consignas del foro fue muchas veces el disparador que accionó debates en la dimensión áulica.

Se definió como eje la perspectiva de Ciudadanía y Convivencia digital (DAE FCNyM, 2021) así como las definiciones de perfiles de usuario seguros (DAE FCNyM, 2021)

Se trabajó desde la inscripción sobre el nombre autopercibido y los aspectos contemplados y no contemplados en los Sistemas de Información. Se tuvo especial cuidado en revisar las matriculaciones en el aula virtual.

SI bien la intervención y su diseño se articulan institucionalmente en virtualidad, visualizamos que contábamos con los recursos y la llegada a la totalidad de la matrícula, esto nos alentó a consolidar las acciones, historizarlas, ordenarlas, secuenciarlas y avanzar en una estrategia que pueda ser narrada, en el desafío de abordar un ingreso virtual, con perspectiva de género, en el cual nos vimos en la situación de proyectarnos con una perspectiva transversal que comprometa al género y las violencias desde el compromiso de todos los actores institucionales. 
SI bien la intervención y su diseño se articulan institucionalmente en virtualidad, visualizamos que contábamos con los recursos y la llegada a la totalidad de la matrícula, esto nos alentó a consolidar las acciones, historizarlas, ordenarlas, secuenciarlas y avanzar en una estrategia que pueda ser narrada, en el desafío de abordar un ingreso virtual, con perspectiva de género, en el cual nos vimos en la situación de proyectarnos con una perspectiva transversal que comprometa al género y las violencias desde el compromiso de todos los actores institucionales. 


\section{BIBLIOGRAFÍA}

Dippolito A., C. Landini y P. Posadas. (2017). Transitar la universidad: implementación de una propuesta de evaluación continua, diagnóstico y acompañamiento. En Inclusión, trayectorias estudiantiles y políticas académicas en la universidad. Compiladoras: Ros, M., Benito, L., Germain, L., Justianovich, S. 173-190 pp disponible en http://sedici.unlp.edu.ar/handle/10915/6

Dippolito A., (2020). ¿Cómo se organiza un curso virtual? Docentes en Línea FAHCE, UNLP. https://www.academia.edu/43782990/C\%C3\%B3

Lago F.y A. Dippolito. (2016). El curso introductorio de la FCNYM: experiencia mediante aulas web en el módulo de Biología. Encuentro Nacional de Servicios de Orientación Universitaria. "Hacia una mirada interdisciplinaria". UNLP. Actas. Disponible en http://blogs.unlp.edu.ar/encuentroorientacionuniversitaria/files/2016/11/ponencias_estrategias_de_ingreso.pdf

Ottaviano, C (2020). Derecho humano a la comunicación: desconcentración, diversidad e inclusión. UNDAVE Ediciones.

Untoiglich, G. et al. (2013). En la infancia los diagnósticos se escriben con lápiz. Buenos Aires. Noveduc. Colección conjunciones. Tomo 33. Pág. 62-63. ISBN 978-987-538-373-9

\section{OTROS DOCUMENTOS}

Proyecto Nexos UNLP, Cap 2 Género. (2018) Dir de inclusión educativa y articulación UNLP. CIN, RENAU y TVU. Dippolito asesora de contenidos, Juan Martín Luis participación. https://www.youtube.com/watch?v=_CFRglpgTkg

Recomendaciones para redactar correo electrónico y conformar un perfil de usuario. (2021). Dirección de Asuntos Estudiantiles FCNYM, UNLP. https:// entornos-educativos.fcnym.unlp.edu.ar/media/attachments/2021/04/09/correo-electronico-y-perfil-de-usuario-2.pdf

Ciudadanía y Convivencia Digital. (2021) Dirección de Asuntos Estudiantiles FCNyM, UNLP. https://entornos-educativos.fcnym.unlp.edu.ar/images/ingreso-2021_ciudadania_digital.pdf 